\title{
LARGE-AREA TRANSITION RADIATION DETECTORS*) FOR ELECTRON IDENTIFICATION IN THE UA6 EXPERIMENT AT THE CERN p̄ COLLIDER
}

\author{
A. Vacchi $^{* *}$ \\ CERN, Geneva, Switzerland
}

\begin{abstract}
Two transition radiation detectors (TRDs) to provide electron-pion discrimination have been built for the UA6 spectrometer at the CERN p $\bar{p}$ Collider. The sensitive area is $(1152 \times 778) \mathrm{mm}$. The $\mathrm{X}$-ray detector is a proportional chamber with a $10 \mathrm{~mm}$ cathode-anode gap and a thin entrance window. The radiator consists of $\mathrm{Li}$ foils in a $\mathrm{He}$ atmosphere. The chamber uses a fully recycled $\mathrm{He}+\mathrm{Xe}+\mathrm{CO}_{2}$ gas mixture. The gas system is remotely controlled. Stable working conditions have been achieved over long periods. The first results agree with the expected performance.
\end{abstract}

To appear in the Proceedings of the Wire Chamber Conference, Vienna, 25-28 February 1986

\footnotetext{
*) Research supported in part by the US Department of Energy.
}

**) Also at Physics Dept., Rockefeller University, New York, NY, USA. 


\section{INTRODUCTION}

An internal hydrogen jet target in the Super Proton Synchrotron (SPS) main ring is used by the CERN UA6 experiment [1] to study final states from antiproton-proton and proton-proton collisions. Each of the two arms of the spectrometer, shown in fig. 1, is equipped with a transition radiation detector (TRD). This is a single lithium radiator-detector element, optimized for electronhadron discrimination at $\gamma=\mathrm{E} / \mathrm{m}$ values larger than $3 \times 10^{4}$. The expected hadron rejection factor for this configuration is $R \geq 10$ (maintaining over $90 \%$ detection efficiency for electrons), using the integrated charge technique. A major design requirement of such a detector is the transparency to electromagnetic particles; the detector represents less than $4 \%$ of a radiation length.

The TRD, of total length $65 \mathrm{~cm}$, is positioned before the first section of an electromagnetic gas-sampling calorimeter [2] made of sandwiches of lead and proportional tubes. The TRD complements the magnetic spectrometer and the calorimeter in electron tagging in order to measure the production of high-mass electron pairs and single electrons in both $\mathrm{p} \overline{\mathrm{p}}$ and $\mathrm{pp}$ interactions at $\sqrt{\mathrm{s}}=24.3 \mathrm{GeV}$.

\section{DESCRIPTION OF THE DETECTOR}

\subsection{The multiwire proportional chamber}

The characteristics of the chamber, schematically shown in fig. 2, are: $1 \mathrm{~cm}$ anode-cathode gap; $20 \mu \mathrm{m}$ diameter sense wires, $8 \mathrm{~mm}$ pitch, interleaved with $60 \mu \mathrm{m}$ diameter field wires; $60 \mu \mathrm{m}$ diameter cathode wire, $2 \mathrm{~mm}$ pitch. Three sense wires are grouped to a preamplifier installed on the chamber. The bipolar preamplifier output is transmitted, through a $63 \mathrm{~m}$ long twisted-pair cable, to delaying and pulse-shaping amplifiers [3] and then digitizated by a LeCroy 2280, charge ADC system. Each group of wires covers a $2.4 \mathrm{~cm}$ wide vertical strip. The operating high voltage is $-2000 \mathrm{~V}$ on the cathode and $-1000 \mathrm{~V}$ on the field wires. From run to run, changes of the order of $2 \%$ to $20 \%$ were made to the high voltage in order to compensate for gas gain variations.

The thickness of Mylar in the path of the X-rays produced in the radiator is $18 \mu \mathrm{m}(6 \mu \mathrm{m}$ outer window of the radiator, $12 \mu \mathrm{m}$ input window to the chamber). The position of the entrance window to the chamber is under continuous automatic control.

\subsection{The lithium radiator}

The advantage of a lithium radiator in the present set-up is the large yield of produced and detected radiation which allows one radiator-detector set, with analog readout, a rejection $R \geq 10$. This radiator has been optimized for the best performance under the conditions of the UA6 experiment. The spacing and the number of foils have been determined for the $45 \mathrm{~cm}$ length of the radiator. Figure 3 shows the calculated $[4,5]$ amount of energy detected in $9 \mathrm{~mm}$ of Xe, for fixed radiator size, as a function of the spacing between foils, taking account of the TR X-ray absorption in the material between the radiator and the chamber. The lithium radiator consists of 800 pure (99.8\%) lithium foils) $(45 \mu \mathrm{m}$ thick, $500 \mu \mathrm{m}$ pitch) in He gas. The foils are flat and vertical; the mean spacing is obtained by using coated stainless-steel spacers which support the foils. The radiators are enclosed in gas-tight containers, and there is a continuous flow of dry helium between the foils. The radiator and detector are assembled as an integrated unit.

\footnotetext{
*) Foote Mineral Company, Exton, Pa., USA.
} 


\subsection{The gas system}

The gas system is divided into three separate parts:

i) a dry helium flow for the lithium radiator;

ii) a $\mathrm{N}_{2}$ flow in the volume between the radiator and the proportional chambers;

iii) a mixture of $\mathrm{Xe}(40 \%)+\mathrm{He}(40 \%)+\mathrm{CO}_{2}(20 \%)$ in the proportional chambers.

The system of the PCs has the following characteristics:

i) a $100 \%$ closed circuit, gas recycling with absorption of $\mathrm{H}_{2} \mathrm{O}$ and $\mathrm{O}_{2}$;

ii) a pneumatic system for control of the position of the entrance window to the chamber;

iii) all operations, including first filling (through $\mathrm{CO}_{2}$ absorption) and gas analysis, are done from the control room. The gas mixture was replaced once during a period of $\sim 100$ days of running.

\subsection{Monitoring}

During data-taking the linear chain is calibrated, at the beginning of each run (i.e. every day), with pulses injected at the input of the preamplifiers; the results as well as the ADC pedestals are recorded on tape.

The gas mixture gain is continuously monitored using a dedicated channel located on one side of the chamber outside the region of the experimental acceptance. This channel records the signal produced by three small ${ }^{55} \mathrm{Fe}$ sources glued on the entrance window. The typical energy resolution for the $5.9 \mathrm{keV} \mathrm{X}$-ray line ranges between $25 \%$ and $35 \%$ FWHM. The overall linearity was verified with ${ }^{57} \mathrm{Co}(14.4 \mathrm{keV})$ and ${ }^{109} \mathrm{Cd}(22.2 \mathrm{keV})$ (Fig. 4).

The monitoring of the gas composition is done by measuring the amount of He present in the mixture, using a calibrated, gas thermal conductivity detector*). The amount of $\mathrm{CO}_{2}$ in the chambers is measured with an infrared gas analyser ${ }^{* *}$. The presence of $\mathrm{O}_{2}$ pollution is detected by means of a zirconium dioxide probe ${ }^{* * *}$ calibrated in the ppm range.

\section{FIRST RESULTS}

\subsection{The conversion trigger}

To produce an enhanced electron sample, a conversion hodoscope is introduced into the experimental acceptance before the first tracking chamber. Photons convert in a $1 \mathrm{~mm}$ thick lead sheet. The electron pairs are deflected horizontally in the UA6 dipole magnet. The conversion trigger sets the following requirements:

i) the signal from a scintillator hodoscope placed before the lead converter is in anticoincidence with a similar set of scintillators placed behind the converter;

ii) more than one track is required in two sets of scintillators positioned downstream from the magnet (four vertical slabs covering the acceptance);

iii) energy deposition in the calorimeter is required using a hodoscope of scintillation counters placed between the first and second modules of the calorimeter (after eight radiation lengths). The hodoscope consists of seven horizontal strips of scintillator.

\footnotetext{
*) Crowcon Instruments, Ltd., Oxford, UK (model 75TC).

**) Leybold-Heraus, Zurich, Switzerland, Infrared gas analyser BINOS 1.

***) Bosch, Zurich, Switzerland, Lambda Sonde, Datenblatt VDT-C6/2-1.
} 


\subsection{Analysis}

In a first off-line analysis of conversion trigger data, taken during the $\mathrm{p} \overline{\mathrm{p}}$ Collider run of autumn 1985, the calorimeter was employed as a particle identifier to show the higher sensitiveness to electrons of the TRDs.

The UA6 calorimeter is divided into three identical modules of 8 radiation lengths each. A hodoscope of scintillators separating the first and second modules is used to trigger on electromagnetic energy deposition in the calorimeter. A first-order rejection of pions is achieved for this preliminary analysis through cuts based on the longitudinal energy deposition of the showers in the calorimeter. For electrons, the total energy is shared between modules 1 and 2. For those pions which deposit a large amount of energy in the calorimeter, the showers generally begin deeper in the calorimeter.

The pulse height of the TRD strip in spatial coincidence with an energy cluster in the calorimeter is shown in Fig. 5 for two samples of events:

i) a charged-hadron-enriched sample, obtained by requiring low $E_{\text {mod.1 }} / E_{\text {tot }}$ in the calorimeter

(Fig. 5a);

ii) an electron-enriched sample obtained by requiring large $E_{\text {mod.1 }} / E_{\text {tot }}$ (Fig. 5b).

The two distributions clearly differ. The electron-enriched sample shows the transition radiation contribution from electrons peaked at $\sim 12$ units of minimum ionization.

Final results concerning the performance of the detector await cleaner electron and pion TRD pulse-height distributions. However, the present preliminary results suggest that the expected hadron rejection $(R \geq 10)$ and electron efficiency $(\sim 90 \%)$ will be achieved. 


\section{REFERENCES}

[1] J. Antille et al. (UA6 Collaboration), Proposal for the study of $\mathrm{e}^{+} \mathrm{e}^{-}, \gamma, \pi^{0}$, and hyperon production in $\mathrm{p} \overline{\mathrm{p}}$ reactions at $\sqrt{\mathrm{s}}=22.5 \mathrm{GeV}$ using an internal jet target at the SPS, SPSC/P148 (1980).

The UA6 Collaboration: CERN - Univ. Lausanne - Univ. Michigan - Rockefeller Univ. The members are: J. Antille, S. Baumann, A. Bernasconi, J.C. Berney, R. Breedon, L. Camilleri, R.L. Cool, P.T. Cox, L. Dick, M. Duro, E.C. Dukes, B. Gabioud, F. Gaille, J.B. Jeanneret, C. Joseph, W. Kubischta, J.F. Loude, E. Malamud, C. Morel, O.E. Overset, J.P. Perroud, P. Petersen, D. Ruegger, R.W. Rusack, G.R. Snow, G. Sozzi, M.T. Tran, A. Vacchi, G. Valenti and G. Von Dardel.

[2] G. Valenti, 5th Topical Workshop on Proton-Antiproton Collider Physics, Saint-Vincent, 1985, ed. M. Greco (World Scientific, Singapore, 1985), p. 534.

[3] Y. Antille et al., Nucl. Instrum. Methods 217 (1983) 327.

[4] G.M. Garibian et al., Nucl. Instrum. Methods 125 (1975) 133.

[5] W. Struczinski, III. Physikalisches Institut, Technische Hochschule, Aachen, private communication. 


\section{Figure captions}

Fig. 1 Layout of experiment UA6 in the SPS tunnel.

Fig. 2 Cross-section of the TRD proportional chambers.

Fig. 3 Energy calibration and linearity.

Fig. 4 Calculated values for the detected energy as a function of spacing for fixed radiator size $(45 \mathrm{~cm})$. The number of foils is recalculated for each point as $N F=45 /$ spacing.

Fig. 5 Some preliminary pulse-height TRD distribution:
a) selecting pions with the calorimeter;
b) selecting electrons with the calorimeter; 


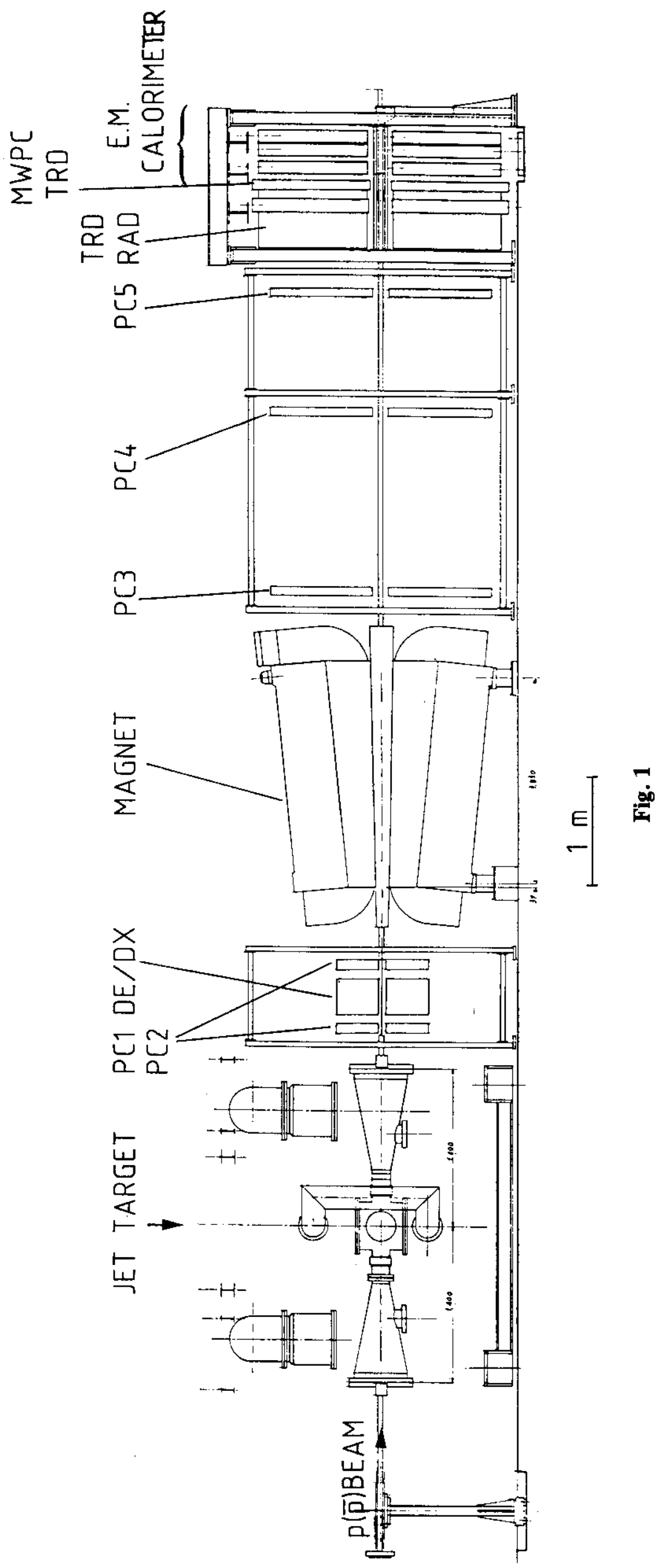




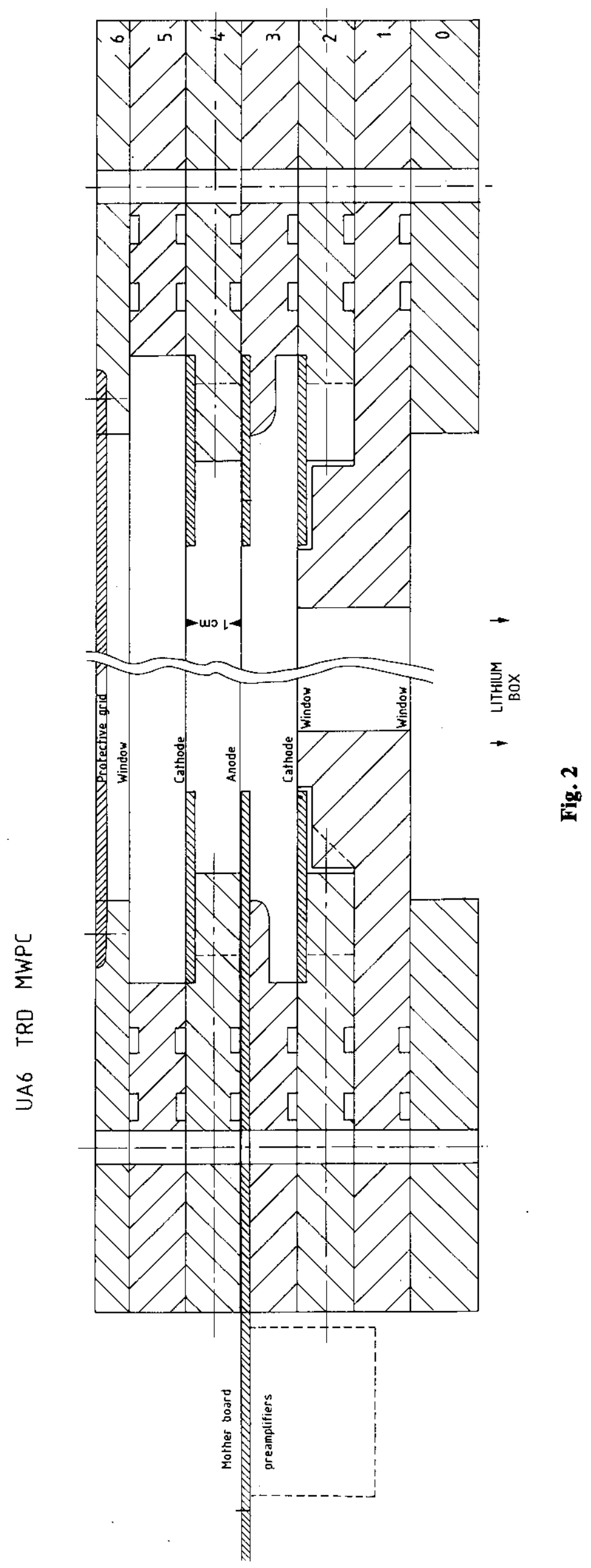




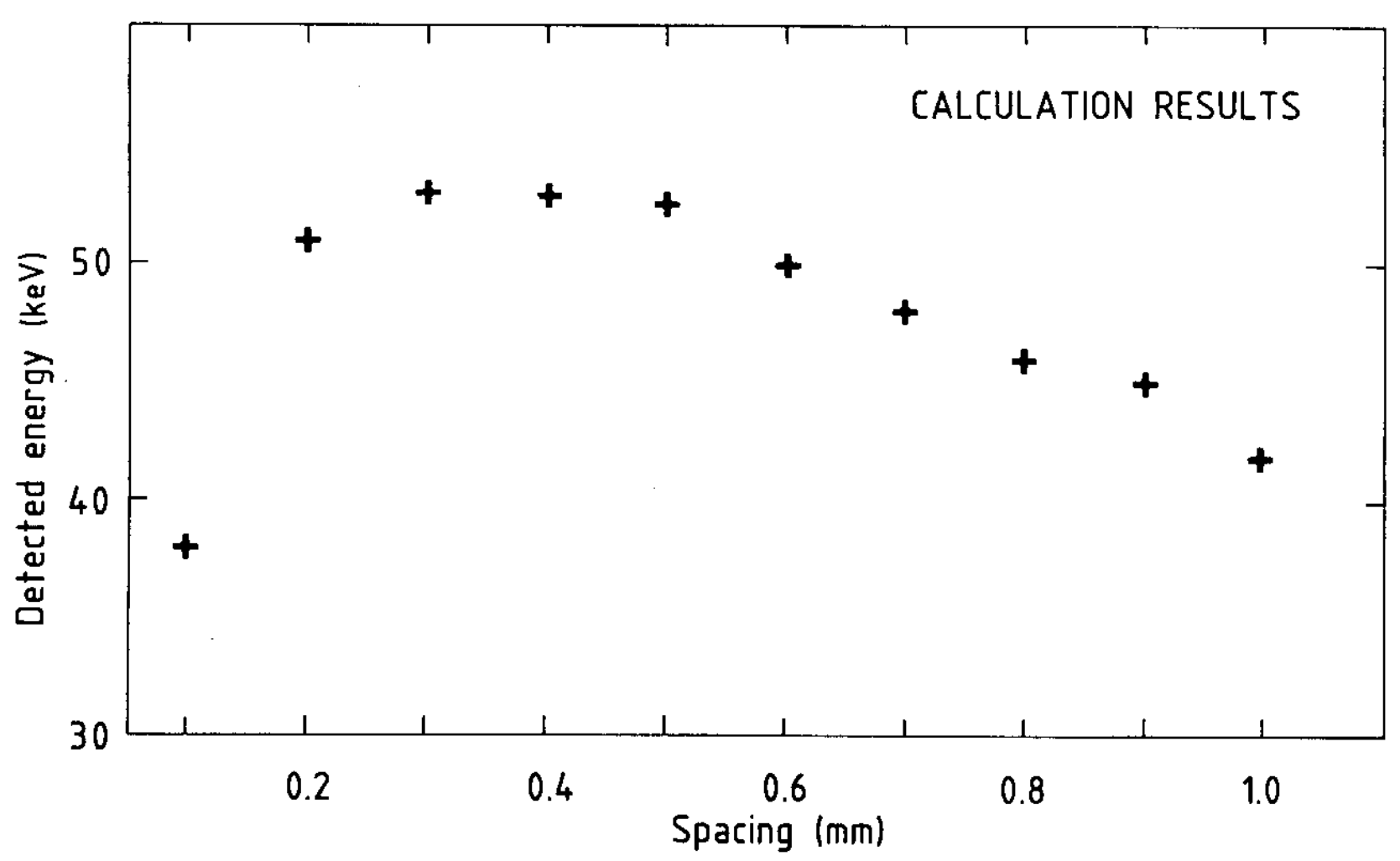

Fig. 3

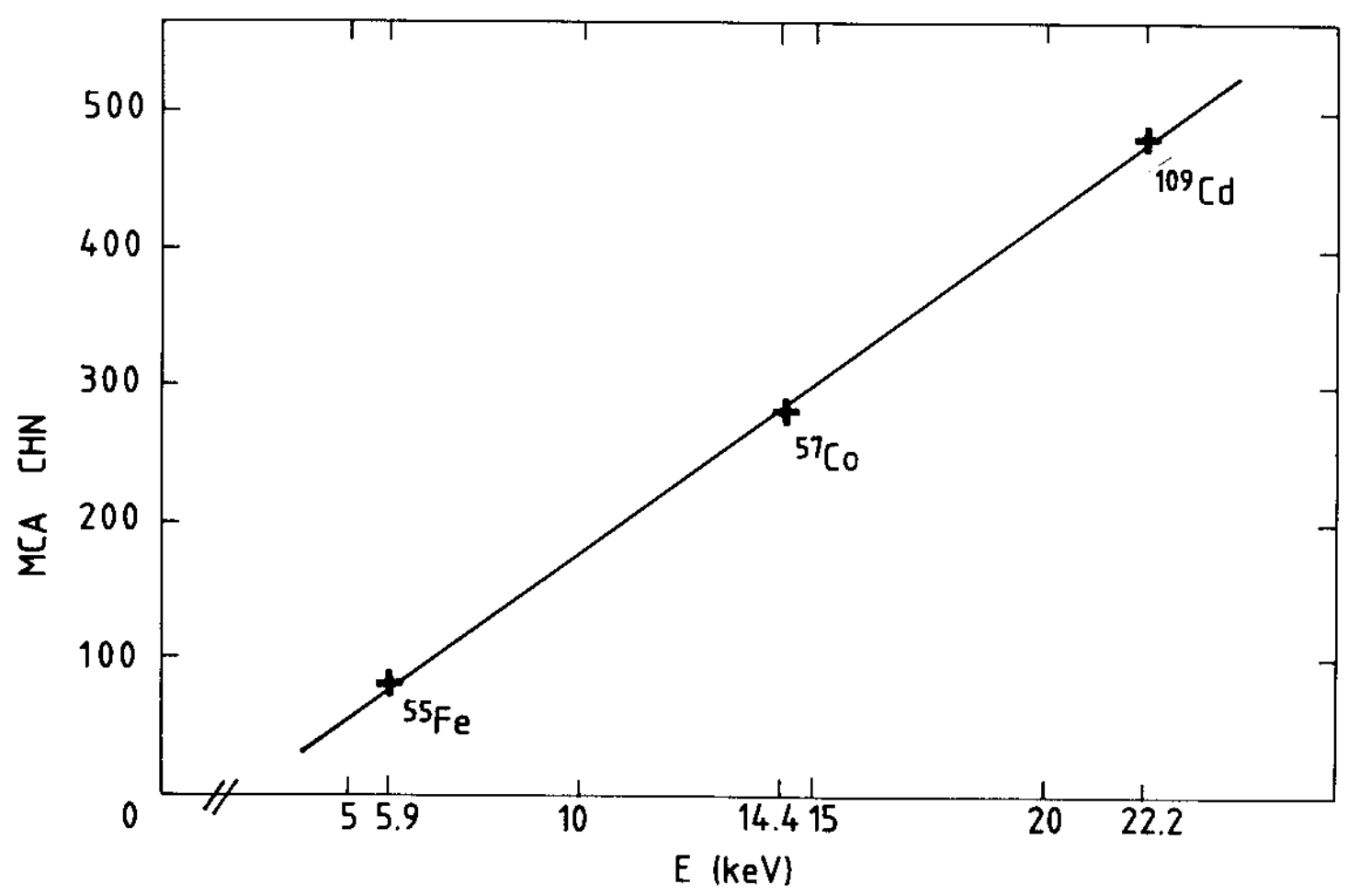

Fig. 4 

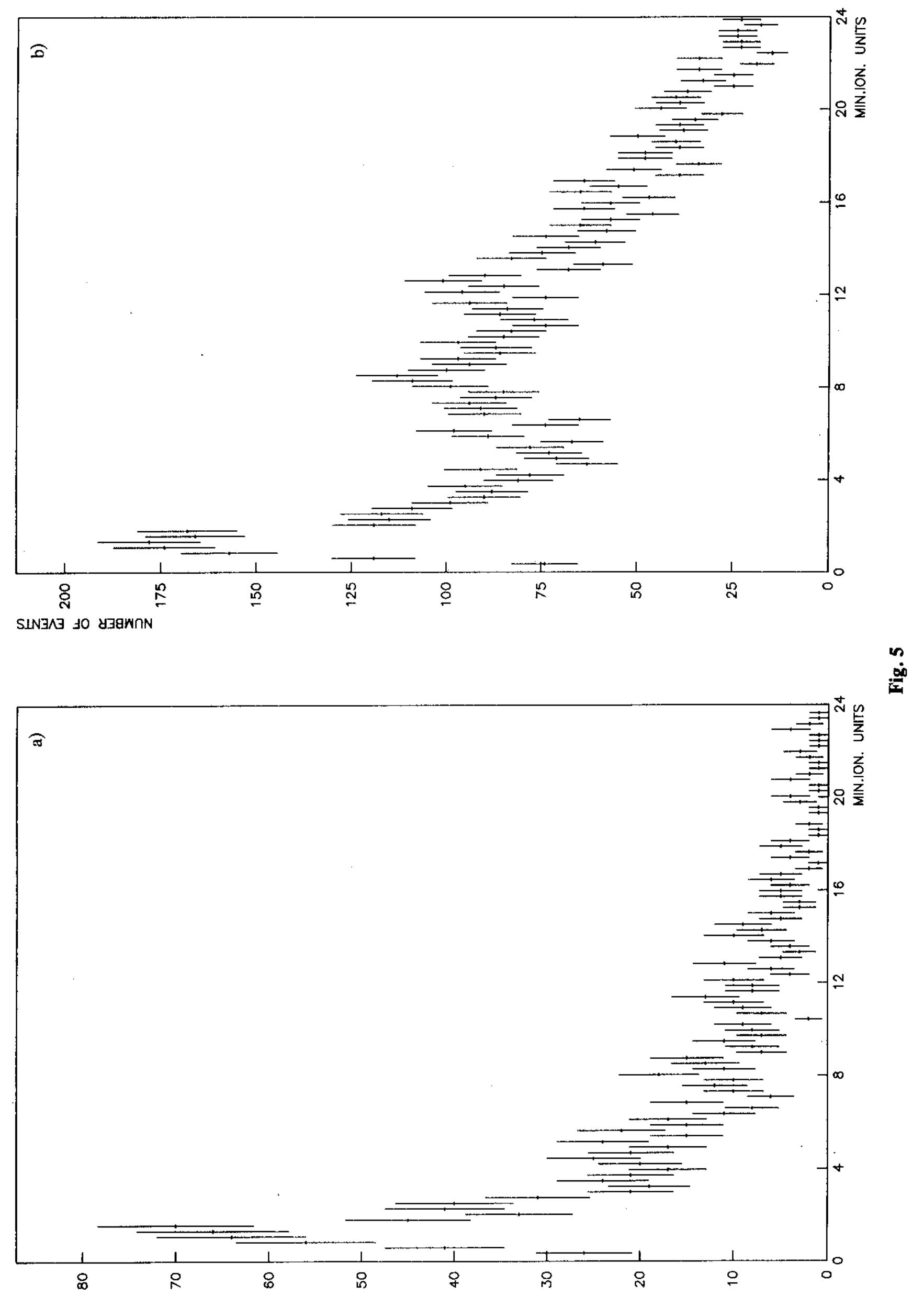